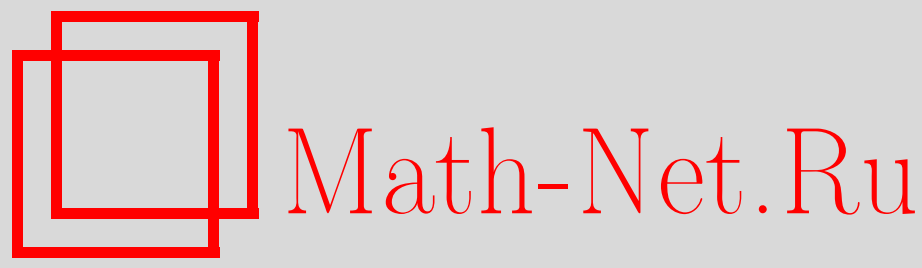

Г. Ф. Ефремов, В. В. Шарков, Квантово-статистическая теория радиационного трения релятивистского электрона, TMФ, 2009, том 158, номер 3, 478-496

DOI: https://doi.org/10.4213/tmf6328

Использование Общероссийского математического портала Math-Net.Ru подразумевает, что вы прочитали и согласны с пользовательским соглашением http://www . mathnet.ru/rus/agreement

Параметры загрузки:

IP : 3.85 .183 .62

26 апреля 2023 г., 07:29:04

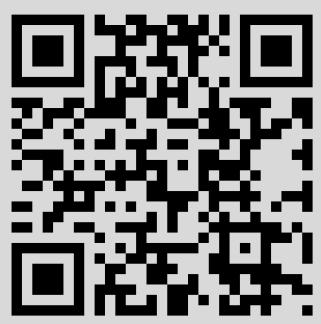




\title{
ФИЗИКА
}

Том 158, № 3

март, 2009

2009 г.

\section{КВАНТОВО-СТАТИСТИЧЕСКАЯ ТЕОРИЯ РАДИАЦИОННОГО ТРЕНИЯ РЕЛЯТИВИСТСКОГО ЭЛЕКТРОНА}

\begin{abstract}
Всесторонне исследован эффект квантовой пространственно-временной нелокальности, учитывающий запаздывание взаимодействия электрона как с собственным полем излучения, так и с флуктуационным полем электромагнитного вакуума. Строго показано, что эффект квантовой нелокальности исключает парадоксы самоускорения и нарушения принципа причинности, присущие классической теории радиационного трения.
\end{abstract}

Ключевые слова: обобщенная радиационная сила трения, теория открытых квантовых систем, квантовая статистика, электромагнитный вакуум, броуновское движение.

\section{1. ВВЕДЕНИЕ}

Проблема радиационного трения и электромагнитной массы электрона возникла до создания квантовой теории в начале XX века. Основополагающий вклад в классическую теорию радиационного трения внесли Абрагам, Лоренц [1] и Дирак [2]. Основные результаты, изложенные, например, в учебниках [3], [4] и монографии [5], активно обсуждаются в литературе на протяжении многих десятилетий (обширная библиография по этому вопросу содержится в [5]).

Классическая формула радиационной силы трения Абрагама-Лоренца [1], [3]-[5]

$$
\mathbf{F}=\frac{2}{3} \frac{e^{2}}{c^{3}} \frac{d^{3}}{d t^{3}} \mathbf{r}(t)
$$

приводит к хорошо известному парадоксу самоускорения [3], [4]: при наличии силы (1) состояние электрона с определенной скоростью оказывается неустойчивым, и электрон за очень короткий промежуток времени $\tau_{0}=10^{-23}$ с приобретает ультрарелятивистскую скорость. Кроме этого, радиационная сила (1) противоречит принципу причинности [6].

Физическая причина парадоксов и расходимостей классической электродинамики обусловлена идеализацией точечности заряженной частицы - электрона. Так как классический электрон не имеет внутренней структуры и является точечным,

${ }^{*}$ Нижегородский государственный университет им. Н. И. Лобачевского, Нижний Новгород, Россия. E-mail: efremov@rf.unn.ru, sharkov@rf.unn.ru 
запаздывание взаимодействия между электроном и его собственным полем излучения отсутствует, вследствие чего и возникают известные парадоксы радиационного трения. Отсутствие запаздывания взаимодействия между электроном и полем излучения приводит также к расходимостям, в классической электродинамике масса электрона оказывается бесконечной.

В последнее время вновь возрос интерес к проблеме радиационного трения. Это относится как к классической, так и к квантовой теории. В классической теории основной интерес представляет радиационное трение в релятивистских задачах [7], [8]. В работе [7] была предложена процедура устранения расходимостей в классической электродинамике и дано точное решение задачи о реакции собственного поля излучения релятивистского точечного электрона. В работе [8], как и в работе [7], рассмотрена реакция излучения при учете перенормировки в классической теории поля с сингулярными источниками. В ряде работ рассматривается реакция излучения в различных моделях классической теории поля (см., например, [9]). Критический анализ существующей классической теории можно найти, например, в работе [10], где также предложены различные модификации этой теории. Попытка устранения парадоксов радиационного трения в рамках классической электродинамики предпринята в работе [11]. Однако несмотря на значительные усилия не удается избежать парадоксов в теории радиационного трения при последовательном классическом способе описания реакции излучения.

Появление квантовой теории не привело к автоматическому устранению парадоксов радиационного трения. Проблема квантовой теории радиационного трения существует на протяжении многих десятилетий и для ее решения предложены разнообразные подходы и приближения [6], [12]-[21]. Один из возможных путей решения проблемы радиационного трения с выходом за рамки теории возмущений основан на методах флуктуационно-диссипационной теории нелинейных открытых квантовых систем [22]-[24]. Эти методы представляют собой принципиальное обобщение линейной теории броуновского движения квантовых систем, развитой Швингером [25] и Сеницким [26]. Эта теория не опирается на предположение марковости и малости взаимодействия. Постановка задачи и исходные уравнения сформулированы в ряде работ как для полевой релятивистской теории [27], так и для одночастичной модели статистической квантовой электродинамики [6].

Полученное в работе [6] строгое квантово-механическое выражение для радиационной силы проанализировано в нерелятивистском пределе и доказано отсутствие парадоксов, присущих классической теории. Квантовая теория радиационного трения для собственного магнитного момента нейтрона предложена в работах [20], [21]. Заметим, что в нерелятивистском пределе исключается вклад в радиационное трение фотонов с большими значениями передаваемого импульса. Однако для полного решения проблемы радиационного трения вклад больших импульсов, передаваемых от поля излучения к электрону, необходимо учитывать.

Как известно, релятивистский электрон имеет внутренние степени свободы, определяемые матрицами Дирака. Наличие внутренней структуры (степеней свободы) у электрона Дирака приводит к установленному в работе [28] эффекту квантовой нелокальности, который явно учитывает запаздывание взаимодействия между электроном и собственным полем излучения. Вследствие этого устраняются парадоксы классической теории радиационного трения. Таким образом, в работе [28] показано, 
ка́к в локальной квантовой теории возникает запаздывание взаимодействия между источником поля (зарядом) и его полем излучения; заметим, что в этой работе рассмотрен лишь один из механизмов радиационного трения, обусловленный реакцией собственного поля излучения.

В настоящей работе исследован чрезвычайно важный релятивистский эффект запаздывания взаимодействия как с собственным полем излучения, так и с флуктуационным полем электромагнитного вакуума. Вследствие этого рассматриваемые явления приобретают особый квантово-статистический характер. Дополнительный учет того факта, что флуктуационное поле электромагнитного вакуума вносит вклад в запаздывание взаимодействия, принципиально важен для исследования радиационных эффектов, имеющих существенно статистическую природу, таких как смещение Лэмба и аномальный магнитный момент электрона, поскольку запаздывание взаимодействия приводит к исключению расходимостей.

\section{2. ОБОБЩЕННАЯ РАДИАЦИОННАЯ СИЛА ТРЕНИЯ}

Рассмотрим взаимодействие релятивистского электрона с квантовым полем излучения и некоторым внешним потенциальным полем $V(\mathbf{r}, t)$. Для исследования радиационных эффектов целесообразно воспользоваться гейзенберговской картиной движения. Прежде всего отметим, что гейзенберговские уравнения движения для динамических переменных электрона в свободном пространстве допускают точное решение, впервые полученное Шредингером в 1930 г. [29]. Кроме этого, имеют место точные стохастические уравнения для динамических переменных электрона [6]. В гейзенберговской картине движения все динамические переменные рассматриваемой системы явно зависят от времени и входят в уравнения движения равноправным образом. Поэтому исходный гамильтониан системы представи́м в виде

$$
H=c \boldsymbol{\alpha}(t)\left(\mathbf{p}(t)-\frac{e}{c} \mathbf{A}(\mathbf{r}(t), t)\right)+\beta(t) m c^{2}+e A_{0}(\mathbf{r}(t), t)+V(\mathbf{r}(t), t)+F(t),
$$

где $F(t)$ - гамильтониан квантового поля излучения, $A_{0}(\mathbf{r}(t), t)$ и $\mathbf{A}(\mathbf{r}(t), t)$ - потенциалы поля излучения, $\boldsymbol{\alpha}(t)$ и $\beta(t)$ - матрицы Дирака, которые подчиняются известным перестановочным соотношениям, например

$$
\alpha_{j}(t) \alpha_{l}(t)+\alpha_{l}(t) \alpha_{j}(t)=2 \delta_{j l}
$$

Распорядимся калибровочной симметрией, выбирая поперечную калибровку для потенциалов поля:

$$
\operatorname{div} \mathbf{A}(\mathbf{r}(t), t)=0
$$

В представлении Гейзенберга потенциалы поля являются функциями координаты электрона $\mathbf{r}(t)$, поэтому их удобно представить в виде разложения Фурье:

$$
\begin{aligned}
& A_{0}(\mathbf{r}(t), t)=\int \frac{d^{3} \mathbf{k}}{(2 \pi)^{3}} e^{i \mathbf{k r}(t)} A_{0}(\mathbf{k}, t), \\
& A_{j}(\mathbf{r}(t), t)=\int \frac{d^{3} \mathbf{k}}{(2 \pi)^{3}} e^{i \mathbf{k r}(t)} A_{j}(\mathbf{k}, t),
\end{aligned}
$$


где фурье-компоненты $A_{j}(\mathbf{k}, t)$ явно не содержат переменных электрона. Канонически сопряженные к $A_{j}(\mathbf{k}, t)$ компоненты плотности тока находим из гамильтониана (2) в соответствии с равенством

$$
-\frac{\delta H}{\delta A_{j}(\mathbf{k}, t)}=\frac{e}{c} \dot{r}_{j}(t) e^{i \mathbf{k r}(t)} .
$$

Запишем уравнения Гейзенберга для динамических переменных рассматриваемой системы. Для фурье-компонент $A_{j}(\mathbf{k}, t)$ потенциалов поля из $(2)$ имеем уравнения

$$
\left(k^{2}+\frac{1}{c^{2}} \frac{d^{2}}{d t^{2}}\right) A_{j}(\mathbf{k}, t)=\frac{4 \pi}{c} e \dot{r}_{j}(t) e^{i \mathbf{k r}(t)},
$$

представляющие собой уравнения Максвелла для поля излучения при взаимодействии с электроном. Запишем также уравнения Гейзенберга для динамических переменных электрона. Из гамильтониана системы (2) следует, в частности, квантовый аналог уравнения Лоренца:

$$
\frac{d}{d t} \pi_{j}(t)+\nabla_{j} V(\mathbf{r}(t), t)+\nabla_{j} A_{0}(\mathbf{r}(t), t)=-\frac{e}{c} \frac{d}{d t} A_{j}(\mathbf{r}(t), t)+\frac{e}{c} \nabla_{j}\left(\dot{r}_{\alpha}(t) A_{\alpha}(\mathbf{r}(t), t)\right)
$$
где

$$
\pi_{j}(t)=p_{j}(t)-\frac{e}{c} A_{j}(\mathbf{r}(t), t)
$$

есть кинетический импульс электрона, а $p_{j}(t)$ - канонический импульс электрона.

Особенность стоящего в правой части (7) квантового аналога силы Лоренца, которая действует на электрон со стороны поля излучения, состоит в том, что эта сила определяется полной производной от векторного потенциала, благодаря чему содержит более простое слагаемое, зависящее от оператора скорости $\dot{r}_{j}(t)=c \alpha_{j}(t)$.

Будем считать, что взаимодействие источника поля (электрона) с полем излучения включается адиабатически медленно в бесконечно удаленный момент времени $t=-\infty$. До включения взаимодействия мы имеем систему невзаимодействующих частиц: электрона в некотором состоянии $\psi \in \Gamma$ и поля излучения в основном состоянии (все числа фотонов $\left.n_{\mathbf{k} s}=0\right)$.

Как известно, в основном, вакуумном, состоянии временна́я функция корреляции потенциалов поля $A_{j}^{0}(\mathbf{k}, t)$ в принятой кулоновской калибровке есть

$$
M_{j l}\left(\mathbf{k}, t-t_{1}\right)=\left\langle\frac{1}{2}\left[A_{j}^{0}(\mathbf{k}, t), A_{l}^{0}\left(-\mathbf{k}, t_{1}\right)\right]_{+}\right\rangle=\frac{\hbar}{2} \frac{4 \pi c}{k} \cos \left(c k\left(t-t_{1}\right)\right)\left(\delta_{j l}-\frac{k_{j} k_{l}}{k^{2}}\right) .
$$

С учетом адиабатического включения взаимодействия решение уравнения (6) имеет вид

$$
A_{j}(\mathbf{k}, t)=A_{j}^{0}(\mathbf{k}, t)+\frac{e}{c} \int_{-\infty}^{\infty} d t_{1} D_{j l}\left(\mathbf{k}, t-t_{1}\right) e^{-i \mathbf{k r}(t)} \dot{r}_{l}\left(t_{1}\right) .
$$

Здесь $A_{j}^{0}(\mathbf{k}, t)$ есть невозмущенное поле электромагнитного вакуума. Второе слагаемое, определяемое функцией Грина фотона $D_{j l}\left(\mathbf{k}, t-t_{1}\right)$, есть собственное поле излучения электрона. В принятой калибровке (3) функции Грина имеют вид [30]

$$
\begin{aligned}
D_{j l}\left(\mathbf{k}, t-t_{1}\right) & =\left\langle\frac{i}{\hbar}\left[A_{j}^{0}(\mathbf{k}, t), A_{l}^{0}\left(-\mathbf{k}, t_{1}\right)\right]_{-}\right\rangle \eta\left(t-t_{1}\right)= \\
& =\frac{4 \pi c}{k} \sin \left(c k\left(t-t_{1}\right)\right)\left(\delta_{j l}-\frac{k_{j} k_{l}}{k^{2}}\right) \eta\left(t-t_{1}\right),
\end{aligned}
$$


где единичная функция Хевисайда $\eta\left(t-t_{1}\right)$ учитывает запаздывание взаимодействия электрона с полем излучения. Компоненты функции Грина $D_{j 0}(\mathbf{k}, \tau)$ и $D_{00}(\mathbf{k}, \tau)$, $\tau=t-t_{1}$, в кулоновской калибровке суть

$$
D_{j 0}(\mathbf{k}, \tau)=0, \quad D_{00}(\mathbf{k}, \tau)=\frac{4 \pi}{k^{2}} \delta(\tau) .
$$

Учитывая приведенное выражение для $D_{00}(\mathbf{k}, \tau)$, легко показать, что скалярный потенциал $A_{0}(\mathbf{r}, t)$ не будет вносить вклад в уравнение $(7)$, и в последующем рассмотрении он будет опущен.

Подставим точное решение (8) в выражение для векторного потенциала (5), определяющее квантовую силу Лоренца в уравнении (7). После симметризации произведения коммутирующих между собой операторов $A_{j}(\mathbf{k}, t)$ и $e^{i \mathbf{k r}(t)}$ получим

$$
\begin{aligned}
A_{j}(\mathbf{r}(t), t)= & \int \frac{d^{3} k}{(2 \pi)^{3}} \frac{1}{2}\left[A_{j}^{0}(\mathbf{k}, t), e^{i \mathbf{k r}(t)}\right]_{+}+ \\
& +\frac{e}{c} \int_{-\infty}^{\infty} d t_{1} \int \frac{d^{3} k}{(2 \pi)^{3}} D_{j l}\left(\mathbf{k}, t-t_{1}\right) \frac{1}{2}\left[e^{i \mathbf{k r}(t)}, \dot{r}_{l}\left(t_{1}\right) e^{-i \mathbf{k r}\left(t_{1}\right)}\right]_{+}
\end{aligned}
$$

Второе слагаемое силы Лоренца в правой части равенства (7) после подстановки решения (8) может быть записано двумя способами:

$$
\begin{gathered}
\frac{e}{c} \nabla_{j}\left(\dot{r}_{\alpha} A_{\alpha}(\mathbf{r}(t), t)\right)=\frac{e}{c} \int \frac{d^{3} k}{(2 \pi)^{3}} i k_{j}\left\{\frac{1}{2}\left[A_{\alpha}^{0}(\mathbf{k}, t), \dot{r}_{\alpha} e^{i \mathbf{k r}(t)}\right]_{+}+\right. \\
\left.+\frac{e^{2}}{c^{2}} \int_{-\infty}^{\infty} d t_{1} D_{\alpha l}\left(\mathbf{k}, t-t_{1}\right) \frac{1}{2}\left[e^{i \mathbf{k r}(t)} \dot{r}_{\alpha}(t), e^{-i \mathbf{k r}\left(t_{1}\right)} \dot{r}_{l}\left(t_{1}\right)\right]_{+}\right\}
\end{gathered}
$$

И

$$
\begin{gathered}
\frac{e}{c} \nabla_{j}\left(\dot{r}_{\alpha} A_{\alpha}(\mathbf{r}(t), t)\right)=\frac{e}{c} \int \frac{d^{3} k}{(2 \pi)^{3}} \nabla_{j}\left\{\frac{1}{2}\left[A_{\alpha}^{0}(\mathbf{k}, t), \dot{r}_{\alpha} e^{i \mathbf{k r}(t)}\right]_{+}+\right. \\
\left.+\frac{e^{2}}{c^{2}} \int_{-\infty}^{\infty} d t_{1} D_{\alpha l}\left(\mathbf{k}, t-t_{1}\right) \frac{1}{2}\left[e^{i \mathbf{k r}(t)} \dot{r}_{\alpha}(t), e^{-i \mathbf{k r}\left(t_{1}\right)} \dot{r}_{l}\left(t_{1}\right)\right]_{+}\right\} .
\end{gathered}
$$

Первое слагаемое в (10) задает параметрическое воздействие флуктуаций вакуумного поля на электрон, которое вносит определенный вклад в динамику наряду со вторым слагаемым в (10) и одновременно включает в себя флуктуационный источник вакуумного поля. Для того чтобы выделить вклад в динамику флуктуаций электромагнитного вакуума, необходимо исключить из уравнения движения электрона вакуумные потенциалы поля. Подобную процедуру исключения фононных переменных при выводе обобщенного кинетического уравнения для электронов проводимости можно найти в работе [24].

В соответствии с методами, предложенными в работе [23], имеем

$$
\begin{aligned}
A_{j}(\mathbf{r}(t), t)= & \frac{e}{c} \int_{-\infty}^{\infty} d t_{1} \int \frac{d^{3} k}{(2 \pi)^{3}}\left\{M_{j l}\left(\mathbf{k}, t-t_{1}\right) \frac{i}{\hbar}\left[e^{i \mathbf{k r}(t)}, \dot{r}_{l}\left(t_{1}\right) e^{-i \mathbf{k r}\left(t_{1}\right)}\right]_{-} \eta\left(t-t_{1}\right)+\right. \\
& \left.+D_{j l}\left(\mathbf{k}, t-t_{1}\right) \frac{1}{2}\left[e^{i \mathbf{k r}(t)}, \dot{r}_{l}\left(t_{1}\right) e^{-i \mathbf{k r}\left(t_{1}\right)}\right]_{+}\right\}+\xi_{j}^{A}(t)
\end{aligned}
$$


где $\xi_{j}^{A}(t)$ - это вклад в общий флуктуационный источник от первого слагаемого в силе трения, которое определяется только векторным потенциалом. Здесь с помощью единичной функции Хевисайда $\eta\left(t-t_{1}\right)$ мы учитываем принцип причинности при параметрическом воздействии на электрон поля электромагнитного вакуума. В соответствии с общей теорией [23] флуктуационные источники $\xi_{j}(t)$ строго определены и для них указан рецепт вычисления функции корреляции любого порядка. В итоге получим уравнение, описывающее броуновское движение релятивистского электрона Дирака в поле электромагнитного вакуума:

$$
\frac{d}{d t} \pi_{j}(t)+\nabla_{j} V(\mathbf{r}, t)=F_{j}(t)+\xi_{j}(t)
$$

где $\xi_{j}(t)$ есть строго определенный флуктуационный источник с равным нулю средним значением по вакуумному состоянию поля. Обобщенная радиационная сила трения $F_{j}(t)$ учитывает как реакцию собственного поля излучения на электрон, так и воздействие флуктуаций вакуумного поля. Строгое выражение для радиационной силы $F_{j}(t)$ в $(14)$ с учетом (11) имеет вид

$$
\begin{aligned}
F_{j}(t)= & -\frac{e^{2}}{c^{2}} \frac{d}{d t} \int_{-\infty}^{\infty} d t_{1} \int \frac{d^{3} k}{(2 \pi)^{3}}\left\{D_{j l}\left(\mathbf{k}, t-t_{1}\right) \frac{1}{2}\left[e^{i \mathbf{k r}(t)}, e^{-i \mathbf{k r}\left(t_{1}\right)} \dot{r}_{l}\left(t_{1}\right)\right]_{+}+\right. \\
& \left.+M_{j l}\left(\mathbf{k}, t-t_{1}\right) \frac{i}{\hbar}\left[e^{i \mathbf{k r}(t)}, e^{-i \mathbf{k r}\left(t_{1}\right)} \dot{r}_{l}\left(t_{1}\right)\right]_{-} \eta\left(t-t_{1}\right)\right\}+ \\
& +\frac{e^{2}}{c^{2}} \int_{-\infty}^{\infty} d t_{1} \int \frac{d^{3} k}{(2 \pi)^{3}} i k_{j}\left\{D_{\alpha l}\left(\mathbf{k}, t-t_{1}\right) \frac{1}{2}\left[e^{i \mathbf{k r}(t)} \dot{r}_{\alpha}(t), e^{-i \mathbf{k r}\left(t_{1}\right)} \dot{r}_{l}\left(t_{1}\right)\right]_{+}+\right. \\
& \left.+M_{\alpha l}\left(\mathbf{k}, t-t_{1}\right) \frac{i}{\hbar}\left[e^{i \mathbf{k r}(t)} \dot{r}_{\alpha}(t), e^{-i \mathbf{k r}\left(t_{1}\right)} \dot{r}_{l}\left(t_{1}\right)\right]_{-} \eta\left(t-t_{1}\right)\right\} .
\end{aligned}
$$

Заметим, что при записи второго слагаемого силы Лоренца в форме (12) необходимо дополнительно учесть спаривание $A_{j}^{0}(\mathbf{k}, t)$ с оператором $\nabla_{j}$, действующим на него слева, которое приведено в одной из работ авторов. Наличие $\nabla_{j}$ в выражении (12) позволяет непосредственно определить перенормировку потенциальной энергии $V(\mathbf{r})$ за счет флуктуаций электромагнитного вакуума и на основе этого вычислить смещение Лэмба. Выражение для радиационной силы в форме (15) оказывается более предпочтительным для всестороннего исследования квантово-механических эффектов радиационного трения.

Точное выражение для радиационной силы (15) включает в себя оба тесно связанных между собой механизма радиационного трения: воздействие на электрон флуктуаций электромагнитного вакуума и воздействие собственного поля излучения. Совместный учет указанных физических механизмов имеет принципиальное значение для исследования на основе формул (14) и (15) различных электродинамических эффектов. Кроме этого, из строгого выражения (15) следует, что среднее значение радиационной силы в состоянии термодинамического равновесия электрона с полем излучения равно нулю. Заметим, что согласно работе [6] выражение (15) справедливо при взаимодействии электрона с тепловым полем излучения при конечной температуре. Чтобы доказать указанное выше свойство, следует записать среднее значение для (15) в спектральной форме и воспользоваться флуктуационно-диссипационной теоремой Каллена-Вельтона. 


\section{3. КОЭФФИЦИЕНТ РАДИАЦИОННОГО ТРЕНИЯ ЭЛЕКТРОНА}

Исследуем особенности эффекта радиационного трения, обусловленные запаздыванием взаимодействия как с собственным полем излучения, так и с флуктуационным полем электромагнитного вакуума.

Пусть на электрон, эволюция во времени которого определяется уравнением (14), действует однородное электрическое поле с напряженностью $\mathbf{E}(t)$, энергия взаимодействия с этим полем есть

$$
V(t)=-r_{j} e E_{j}(t)=-r_{j} f_{j}
$$

Усредним уравнение (14) по начальному состоянию системы и, принимая во внимание равенство нулю среднего значения флуктуационного источника, получим

$$
\frac{d}{d t}\left\langle\pi_{j}(t)\right\rangle=f_{j}(t)+\left\langle F_{j}(t)\right\rangle .
$$

Из этого уравнения определим отклик на внешнюю силу $f_{j}(t)$ в соответствии с тем, что

$$
\left\langle r_{j}(t)\right\rangle=\int d t_{1} \varphi_{j l}\left(t, t_{1}\right) f_{l}\left(t_{1}\right)
$$

откуда находим

$$
\left\langle\left.\frac{\delta r_{j}(t)}{\delta f_{l}\left(t_{2}\right)}\right|_{\mathbf{f}=0}\right\rangle=\varphi_{j l}\left(t, t_{2}\right)=\left\langle\frac{i}{\hbar}\left[r_{j}(t), r_{l}\left(t_{2}\right)\right]_{-}\right\rangle \eta\left(t-t_{2}\right) .
$$

Будем считать, что до включения взаимодействия (16) система находилась в равновесии; как было отмечено выше, среднее значение радиационной силы в равновесии равно нулю. С учетом вышесказанного для среднего значения радиационной силы можно записать

$$
\left\langle F_{j}(t)\right\rangle=\int d t_{2}\left\langle\frac{\delta F_{j}(t)}{\delta f_{l}\left(t_{2}\right)}\right\rangle f_{l}\left(t_{2}\right)
$$

Из выражения(15) для радиационной силы имеем

$$
\left\langle\frac{\delta F_{j}(t)}{\delta f_{l}\left(t_{2}\right)}\right\rangle=\int d t_{1}\left\langle L_{j n}\left(t, t_{1}\right) \frac{\delta r_{n}\left(t_{1}\right)}{\delta f_{l}\left(t_{2}\right)}\right\rangle .
$$

Так как нелинейность системы, связанная с радиационной силой, мала, можно пренебречь флуктуациями отклика в соответствии с нелинейными флуктуационно-диссипационными теоремами [31]. Поэтому в (19) можно заменить отклик его статистическим средним значением:

$$
\left\langle\frac{\delta F_{j}(t)}{\delta f_{l}\left(t_{2}\right)}\right\rangle=\int d t_{1}\left\langle L_{j n}\left(t, t_{1}\right)\right\rangle\left\langle\frac{\delta r_{n}\left(t_{1}\right)}{\delta f_{l}\left(t_{2}\right)}\right\rangle .
$$

Как уже говорилось выше, основной вклад в радиационное трение вносит первое слагаемое в (15), которое мы будем рассматривать в настоящей работе. Приведем явное выражение для радиационной силы (15), опуская второе слагаемое. С этой 
целью подставим в (15) выражение для функции Грина фотона (9) и запаздывающую функцию корреляции $\widetilde{M}_{j l}(\mathbf{k}, \tau)=M_{j l}(\mathbf{k}, \tau) \eta(\tau)$, для которой можно записать следующее соотношение:

$$
\widetilde{M}_{j l}(\mathbf{k}, \tau)=-\frac{1}{c k} \frac{\hbar}{2} \frac{d}{d t_{1}} D_{j l}(\mathbf{k}, \tau) .
$$

После подстановки (22) в (15) с помощью интегрирования по частям производная по времени переносится на оператор скорости. Подобным образом можно поступить и с формулой (9), переписав ее в виде

$$
D_{j l}(\mathbf{k}, \tau)=\frac{4 \pi}{k^{2}} \frac{d}{d t_{1}}(\cos (c k \tau) \eta(\tau))\left(\delta_{j l}-\frac{k_{j} k_{l}}{k^{2}}\right) .
$$

Подставим два последних соотношения в (16) и проинтегрируем по частям по времени, с учетом поперечной калибровки получим

$$
\left\langle\frac{1}{m} F_{j}(t)\right\rangle=\int d t_{1} \gamma_{j l}\left(t-t_{1}\right)\left\langle\dddot{r}_{l}\left(t_{1}\right)\right\rangle,
$$

где коэффициент трения $\gamma_{j l}\left(t-t_{1}\right)$, определяемый формулой $(24)$, есть

$$
\begin{aligned}
\gamma_{j l}\left(t-t_{1}\right)= & \frac{1}{2 \pi^{2}} \frac{e^{2}}{m c^{2}} \int d^{3} k \frac{1}{k^{2}}\left(\delta_{j l}-\frac{k_{j} k_{l}}{k^{2}}\right) \times \\
& \times\left\{\cos \left(c k\left(t-t_{1}\right)\right)\left\langle\frac{1}{2}\left[e^{i \mathbf{k r}(t)}, e^{-i \mathbf{k r}\left(t_{1}\right)}\right]_{+}\right\rangle_{0}-\right. \\
& \left.-\sin \left(c k\left(t-t_{1}\right)\right)\left\langle\frac{i}{2}\left[e^{i \mathbf{k r}(t)}, e^{-i \mathbf{k r}\left(t_{1}\right)}\right]_{-}\right\rangle_{0}\right\} \eta\left(t-t_{1}\right) .
\end{aligned}
$$

Здесь скобки $\langle\cdot\rangle_{0}$ означают среднее по невозмущенному состоянию полной системы. В силу изотропии начального состояния системы средние значения коммутатора и антикоммутатора экспоненциальных множителей в (25) не зависят от направления волнового вектора $\mathbf{k}$. Следовательно, $\gamma_{j l}\left(t-t_{1}\right)=\delta_{j l} \gamma\left(t-t_{1}\right)$, где

$$
\begin{aligned}
\gamma\left(t-t_{1}\right)= & \frac{4}{3 \pi} \frac{e^{2}}{m c^{2}} \int_{0}^{\infty} d k\left\{\cos \left(c k\left(t-t_{1}\right)\right)\left\langle\frac{1}{2}\left[e^{i \mathbf{k r}(t)}, e^{-i \mathbf{k r}\left(t_{1}\right)}\right]_{+}\right\rangle_{0}-\right. \\
& \left.-\sin \left(c k\left(t-t_{1}\right)\right)\left\langle\frac{i}{2}\left[e^{i \mathbf{k r}(t)}, e^{-i \mathbf{k r}\left(t_{1}\right)}\right]_{-}\right\rangle_{0}\right\} \eta\left(t-t_{1}\right) .
\end{aligned}
$$

Заметим, что в классическом пределе коэффициент трения принимает вид

$$
\gamma\left(t-t_{1}\right)=\gamma_{0} \delta\left(t-t_{1}\right)=\frac{2}{3} \frac{e^{2}}{m c^{3}} \delta\left(t-t_{1}\right),
$$

что согласуется с классической формулой (1).

Коэффициент радиационного трения (26) имеет существенно нелинейную зависимость от переменных электрона $\mathbf{r}(t)$ и $\mathbf{r}\left(t_{1}\right)$, взятых в различные моменты времени. Благодаря этому квантово-механическое выражение учитывает запаздывание взаимодействия между электроном и полем излучения. Для иллюстрации этого 
вычислим линейную восприимчивость электрона из уравнения (17) с учетом запаздывания взаимодействия, которое определяется силой радиационного трения, задаваемой формулами (24)-(26). С этой целью перепишем (17) в собственной системе координат:

$$
\frac{d^{2}}{d t^{2}}\left\langle r_{j}(t)\right\rangle=\frac{1}{m} f_{j}(t)+\int d t_{1} \gamma\left(t-t_{1}\right) \frac{d^{3}}{d t_{1}^{3}}\left\langle r_{j}\left(t_{1}\right)\right\rangle .
$$

Из этого уравнения определим отклик на гармоническую силу $f_{j}(t)=f_{j}(\omega) e^{-i \omega t}$. По определению имеем

$$
\left\langle r_{j}(\omega)\right\rangle=\chi(\omega) f_{j}(\omega)
$$

Применим к (28) преобразование Фурье, получим

$$
-\omega^{2}\left\langle r_{j}(\omega)\right\rangle=\frac{1}{m} f_{j}(\omega)+i \omega^{3} \gamma(\omega)\left\langle r_{j}(\omega)\right\rangle
$$

В соответствии с (29) линейная восприимчивость будет иметь следующий вид:

$$
\chi(\omega)=-\frac{1}{m} \frac{1}{\omega^{2}(1+i \omega \gamma(\omega))} .
$$

Согласно принципу причинности восприимчивость должна быть аналитической функцией в верхней полуплоскости комплексной переменной $\omega$, в то же время из классической формы (27) вместо (30) имеем выражение для восприимчивости с полюсом в верхней полуплоскости комплексной переменной $\omega$,

$$
\chi(\omega)=-\frac{1}{m} \frac{1}{\omega^{2}\left(1+i \omega \gamma_{0}\right)},
$$

что противоречит принципу причинности.

\section{4. ЭФФЕКТ ПРОСТРАНСТВЕННО-ВРЕМЕННОЙ КВАНТОВОЙ НЕЛОКАЛЬНОСТИ}

В отличие от классического коэффициента (27) квантовомеханический коэффициент радиационного трения (26) автоматически учитывает принцип причинности и запаздывание взаимодействия электрона с полем излучения, связанное с конечностью скорости распространения света и некоммутативностью гейзенберговских операторов координаты электрона, взятых в различные моменты времени.

Принципиально нелинейная и нелокальная зависимость радиационной силы от операторов координаты $\mathbf{r}(t)$ и $\mathbf{r}\left(t_{1}\right)$ определяется произведением экспоненциальных множителей, которое может быть записано в виде

$$
e^{i \mathbf{k r}(t)} e^{-i \mathbf{k r}\left(t_{1}\right)}=e^{i \mathbf{k} \Delta \mathbf{r}} e^{-i B},
$$

где $\Delta \mathbf{r}=\mathbf{r}(t)-\mathbf{r}\left(t_{1}\right)$ есть оператор приращения координаты за промежуток времени $t-t_{1}$. Наличие в (31) унитарного оператора $e^{-i B}$ обусловлено некоммутативностью операторов $\mathbf{r}(t)$ и $\mathbf{r}\left(t_{1}\right)$ в различные моменты времени и определенным образом влияет на запаздывание взаимодействия между электроном и полем излучения.

В настоящей работе мы учтем вклад в радиационное трение, вносимый большими передаваемыми импульсами. В этом случае асимптотический $(k \rightarrow \infty)$ вклад 
в (26) соответствует малым значениям $\Delta \mathbf{r}$ и, следовательно, малым промежуткам времени. Благодаря этому при вычислении асимптотики коэффициента радиационного трения (26) достаточно воспользоваться приближением свободной эволюции для оператора координаты электрона $r_{j}(t)$; эта эволюция определяется гамильтонианом

$$
H=c \boldsymbol{\alpha} \mathbf{p}+\beta \varepsilon_{0}, \quad \varepsilon_{0}=m c^{2} .
$$

Из уравнений Гейзенберга имеем

$$
\dot{r}_{j}(t)=c \alpha_{j}, \quad \dot{\alpha}_{j}(t)=\frac{1}{i \hbar}\left[\alpha_{j}, H\right] .
$$

Отметим, что импульс р и гамильтониан электрона (32) являются интегралами движения. Из перестановочных соотношений для матриц Дирака $\boldsymbol{\alpha}$ и $\beta$ имеем также соотношения

$$
\alpha_{j} H+H \alpha_{j}=2 c p_{j} .
$$

Решая систему уравнений (33) с учетом (34), находим

$$
\begin{aligned}
r_{j}(t)= & r_{j}(0)+\frac{c^{2} p_{j}}{H}\left(t-\frac{\hbar}{2 \widetilde{H}} \sin \left(\frac{2 \widetilde{H}}{\hbar} t\right)\right)+\alpha_{j}(0) \frac{\hbar c}{2 \widetilde{H}} \sin \left(\frac{2 \widetilde{H}}{\hbar} t\right)+ \\
& +\dot{\alpha}_{j}(0) \frac{\hbar^{2} c}{4 \widetilde{H}^{2}}\left(1-\cos \left(\frac{2 \widetilde{H}}{\hbar} t\right)\right)
\end{aligned}
$$

где $\widetilde{H}^{2} \equiv H^{2}=c^{2} p^{2}+\varepsilon_{0}^{2}$ и, следовательно, $\widetilde{H}=\sqrt{c^{2} p^{2}+\varepsilon_{0}^{2}}$. Впервые такое решение было получено Шредингером [29].

Как и в работе [28], воспользуемся решением (35) для вычисления дисперсии оператора

$$
F_{0}=\mathbf{k}\left(\mathbf{r}(t)-\mathbf{r}\left(t_{1}\right)\right)=\mathbf{k} \Delta \mathbf{r}
$$

в собственной системе координат электрона. Используя перестановочные соотношения для матриц $\alpha_{j}$ и $\dot{\alpha}_{j}$, получим следующее выражение для дисперсии приращения (36):

$$
F_{0}^{2}=k^{2} \lambda_{\mathrm{c}}^{2} \sin ^{2}\left(\omega_{0} t\right)=x^{2} \sin ^{2}\left(\omega_{0} t\right),
$$

где $\omega_{0}=m c^{2} / \hbar, \lambda_{c}=1 / k_{0}$ - комптоновская длина волны фотона, $x=k / k_{0}$. Дисперсия приращения координаты электрона Дирака в собственной системе координат оказывается конечной в отличие от случая релятивистской бесспиновой частицы. Быстрые осцилляции на больших временах, т.е. при $t \gg \tau_{0}=1 / \omega_{0}$, оказываются несущественными, и можно считать, что

$$
F_{0}^{2} \approx \frac{1}{2} k^{2} \lambda_{\mathrm{c}}^{2}, \quad \Delta r \approx \frac{1}{\sqrt{2}} \lambda_{\mathrm{c}} .
$$

Таким образом, дисперсия приращения координаты $\mathbf{r}(t)-\mathbf{r}\left(t_{1}\right)$ для электрона Дирака оказывается конечной в собственной системе координат благодаря наличию собственных (внутренних) степеней свободы, задаваемых матрицами Дирака. Конечное значение дисперсии, как будет показано ниже, приводит к запаздыванию взаимодействия между электроном и собственным полем излучения, что устраняет известные парадоксы радиационного трения и логарифмическую расходимость 
в квантовой электродинамике. Запаздывание определяется временем прохождения светом комптоновской длины волны электрона

$$
\tau_{0}=\frac{\lambda_{0}}{c}=\frac{\hbar}{m c^{2}}=\frac{1}{\omega_{0}} .
$$

Следовательно, квантовая теория радиационного трения должна быть принципиально немарковской теорией, учитывающей запаздывание взаимодействия между электроном и полем излучения. Так как характерное время запаздывания взаимодействия (38) определяется энергией покоя $m c^{2}$, квантовая теория оказывается принципиально релятивистской теорией.

\section{5. ВЫЧИСЛЕНИЕ АСИМПТОТИЧЕСКОГО ВЫРАЖЕНИЯ ДЛЯ КОЭФФИЦИЕНТА РАДИАЦИОННОГО ТРЕНИЯ ЭЛЕКТРОНА В РЕЛЯТИВИСТСКОМ СЛУЧАЕ}

Приведем строгое обоснование эффекта квантовой нелокальности, учитывающего запаздывание взаимодействия электрона как с собственным полем излучения, так и с флуктуационным полем электромагнитного вакуума. Для этого найдем коэффициент радиационного трения в асимптотическом пределе $k \gg k_{0}$. Чтобы вычислить коммутатор и антикоммутатор в (26), запишем произведение экспонент в виде $(31)$, где множитель $e^{i \mathbf{k} \Delta \mathbf{r}}$, который можно точно вычислить, пользуясь соотношением (35), содержит, в частности, пространственную нелокальность $\Delta r$ из (37). Поскольку в оператор $\Delta \mathbf{r}$ согласно формуле (35) входят матрицы Дирака, можно представить $e^{i \mathbf{k} \Delta \mathbf{r}}$ в виде

$$
e^{i \mathbf{k} \Delta \mathbf{r}}=\cos (k \Delta r)+\frac{i \mathbf{k} \Delta \mathbf{r}}{k \Delta r} \sin (k \Delta r),
$$

где $\Delta r=|\Delta \mathbf{r}|=\lambda_{0}\left|\sin \left(\omega_{0} \tau\right)\right|$ не содержит матриц Дирака.

Особенности вычисления унитарного оператора $e^{-i B}$ обусловлены зависимостью координаты электрона $\mathbf{r}(t)$ от импульса и матриц Дирака (см. формулу (35)). В результате вычислений на основе теории $S$-матрицы, приведенных в приложении, имеем

$$
\begin{aligned}
e^{i \mathbf{k r}(t)} e^{-i \mathbf{k r}\left(t_{1}\right)} & =e^{i \mathbf{k} \Delta \mathbf{r}} e^{-i \omega_{0} \tau(\beta x \operatorname{arctg} x+\mathbf{n} \boldsymbol{\alpha}(x-\operatorname{arctg} x)) / 2}, \\
e^{-i \mathbf{k r}\left(t_{1}\right)} e^{i \mathbf{k r}(t)} & =\left.\left(e^{i \mathbf{k r}(t)} e^{-i \mathbf{k r}\left(t_{1}\right)}\right)^{\dagger}\right|_{\mathbf{k} \rightarrow-\mathbf{k}}= \\
& =e^{-i \omega_{0} \tau(-\beta x \operatorname{arctg} x+\mathbf{n} \boldsymbol{\alpha}(x-\operatorname{arctg} x)) / 2} e^{i \mathbf{k} \Delta \mathbf{r}},
\end{aligned}
$$

где $\mathbf{n}-$ единичный вектор в направлении $\mathbf{k}, \tau=t-t_{1}, k=k_{0} x$. Найденные выражения позволяют записать в явном виде коммутатор и антикоммутатор, входящие в формулу (26) для коэффициента радиационного трения.

Учтем формулы (39), (40) и вытекающее из (35) представление $\Delta \mathbf{r}$ в собственной системе координат, после усреднения по основному состоянию получим

$$
\begin{aligned}
\left\langle\frac{1}{2}\left[e^{i \mathbf{k r}(t)}, e^{-i \mathbf{k r}\left(t_{1}\right)}\right]_{+}\right\rangle & =\cos \left(x\left|\sin \left(\omega_{0} \tau\right)\right|\right) \cos \left(\frac{\omega_{0} \tau}{2} f(x)\right), \\
\left\langle\frac{i}{2}\left[e^{i \mathbf{k r}(t)}, e^{-i \mathbf{k r}\left(t_{1}\right)}\right]_{-}\right\rangle & =\frac{x \operatorname{arctg} x}{f(x)} \cos \left(x\left|\sin \left(\omega_{0} \tau\right)\right|\right) \sin \left(\frac{\omega_{0} \tau}{2} f(x)\right),
\end{aligned}
$$


где $f(x)=\sqrt{(x-\operatorname{arctg} x)^{2}+x^{2} \operatorname{arctg}^{2} x}$. После подстановки равенств (41) в (26) и простейших тригонометрических преобразований коэффициент радиационного трения запишется в виде $\left(\tau=t-t_{1}\right)$

$$
\begin{aligned}
\gamma(\tau)= & \frac{2}{3 \pi} \alpha \int_{0}^{\infty} d x \cos \left(x \sin \left(\omega_{0} \tau\right)\right) \eta(\tau) \times \\
& \times\left\{\left[\cos \left(\omega_{0} \tau\left(x+\frac{f(x)}{2}\right)\right)+\cos \left(\omega_{0} \tau\left(x-\frac{f(x)}{2}\right)\right)\right]+\right. \\
& \left.+\frac{x \operatorname{arctg} x}{f(x)}\left[\cos \left(\omega_{0} \tau\left(x+\frac{f(x)}{2}\right)\right)-\cos \left(\omega_{0} \tau\left(x-\frac{f(x)}{2}\right)\right)\right]\right\} .
\end{aligned}
$$

В выражении для силы трения вклады от каждого из двух механизмов радиационного трения (самовоздействия и влияния вакуума) складываются, поэтому асимптотику обоих слагаемых можно рассмотреть совместно. С этой целью отметим, что особенности $\gamma(\tau)$ в асимптотическом пределе определяются функцией $f(x)$, которую мы запишем как

$$
\begin{gathered}
f(x)=\sqrt{x^{2} y^{2}+(x-y)^{2}}=x F(y), \quad y=\operatorname{arctg} x \leqslant \frac{\pi}{2}, \\
F(y)=\sqrt{y^{2}+\left(1-\frac{y}{\operatorname{tg} y}\right)^{2}} .
\end{gathered}
$$

Основной вклад в асимптотическое выражение для коэффициента радиационного трения вносит область $x>1$, в которой функция $F(y)=F(\operatorname{arctg} x)$ очень медленно меняется и ограничена значением $F(\pi / 2)$. Поэтому определим среднее значение этой функции

$$
\bar{F}=\frac{2}{\pi} \int_{0}^{\pi / 2} d y F(y) \approx 0.85
$$

и положим $f(x)=x \bar{F}$. Так как функция $\frac{x \operatorname{arctg} x}{f(x)}=\frac{y}{F(y)}$ также меняется очень медленно, будем использовать ее среднее значение

$$
\xi=\frac{2}{\pi} \int_{0}^{\pi / 2} d y \frac{y}{F(y)} \approx 0.95
$$

Принимая во внимание (43) и (44), перепишем (42) в виде

$$
\gamma(\tau)=\frac{4}{3 \pi} \alpha \int_{0}^{\infty} d x \cos \left(x \sin \left(\omega_{0} \tau\right)\right) \eta(\tau)\left\{\frac{1+\xi}{2} \cos \left(\beta_{1} x \omega_{0} \tau\right)+\frac{1-\xi}{2} \cos \left(\beta_{2} x \omega_{0} \tau\right)\right\}
$$

где $\beta_{1}=1+\bar{F} / 2 \approx 1.425, \beta_{2}=1-\bar{F} / 2 \approx 0.575$. Выполняя в (45) интегрирование по $x$, получим

$$
\begin{aligned}
\gamma(\tau)= & \frac{4}{3 \pi} \alpha\left\{\frac{1+\xi}{4} \pi\left[\delta\left(\beta_{1} \omega_{0} \tau+\sin \left(\omega_{0} \tau\right)\right)+\delta\left(\beta_{1} \omega_{0} \tau-\sin \left(\omega_{0} \tau\right)\right)\right]+\right. \\
& \left.+\frac{1-\xi}{4} \pi\left[\delta\left(\beta_{2} \omega_{0} \tau+\sin \left(\omega_{0} \tau\right)\right)+\delta\left(\beta_{2} \omega_{0} \tau-\sin \left(\omega_{0} \tau\right)\right)\right]\right\} \eta(\tau) .
\end{aligned}
$$


Учитывая значения параметров $\beta_{1}$ и $\beta_{2}$ и принимая во внимание принцип причинности при $\tau>0$, получим следующее выражение:

$$
\gamma(\tau)=\frac{1}{3} \alpha(1-\xi) \delta\left(\beta_{2} \omega_{0} \tau-\sin \left(\omega_{0} \tau\right)\right) \eta(\tau)
$$

Решая трансцендентное уравнение $\beta_{2} \omega_{0} \tau-\sin \left(\omega_{0} \tau\right)=0$, для его корня $\tau^{*}$ получим $\omega_{0} \tau^{*} \approx 1.72, \sin \left(\omega_{0} \tau^{*}\right)=\sigma \approx 0.99$. Отсюда следует, что замена $\sin \left(\omega_{0} \tau\right) \rightarrow \sigma$ не меняет асимптотического предела для коэффициента трения

$$
\gamma(\tau)=\frac{1}{3} \alpha(1-\xi) \delta\left(\beta_{2} \omega_{0}\left(\tau-\tau^{*}\right)\right) \eta(\tau)
$$

Соответствующая замена $\cos \left(x \sin \left(\omega_{0} \tau\right)\right) \rightarrow \cos (\sigma x)$ в (42), вытекающая из асимптотического предела (47), приводит к выражению, в котором одновременно учитывается асимптотика малых передаваемых импульсов и, соответственно, малых частот:

$$
\begin{aligned}
\gamma(\tau)= & \frac{2}{3 \pi} \alpha \int_{0}^{\infty} d x \cos (\sigma x) \eta(\tau) \times \\
& \times\left\{\left[\cos \left(\omega_{0} \tau\left(x+\frac{f(x)}{2}\right)\right)+\cos \left(\omega_{0} \tau\left(x-\frac{f(x)}{2}\right)\right)\right]+\right. \\
& \left.+\frac{x \operatorname{arctg} x}{f(x)}\left[\cos \left(\omega_{0} \tau\left(x+\frac{f(x)}{2}\right)\right)-\cos \left(\omega_{0} \tau\left(x-\frac{f(x)}{2}\right)\right)\right]\right\} .
\end{aligned}
$$

Действительно, в области малых частот можно пренебречь быстрыми осцилляциями в дисперсии $\cos \left(x \sin \left(\omega_{0} \tau\right)\right)$ приращения координаты в выражении (42).

Таким образом, установленная нами асимптотика в области больших передаваемых импульсов будет одновременно учтена во всей области значений $k$, если в исходном выражении для радиационной силы (15) произвести замены

$$
\begin{aligned}
D_{j l}(\mathbf{k}, \tau) & \rightarrow \widetilde{D}_{j l}(\mathbf{k}, \tau)=D_{j l}(\mathbf{k}, \tau) \cos \left(\sigma \lambda_{\mathrm{c}} k\right), \\
M_{j l}(\mathbf{k}, \tau) \rightarrow \widetilde{M}_{j l}(\mathbf{k}, \tau) & =M_{j l}(\mathbf{k}, \tau) \cos \left(\sigma \lambda_{\mathrm{c}} k\right) .
\end{aligned}
$$

$\mathrm{C}$ физической точки зрения наличие множителя $\cos (\sigma x)$ в (15) согласно (49) приводит к учету запаздывания взаимодействия между источником поля, электроном, и полем излучения. При этом время запаздывания взаимодействия есть время прохождения светом комптоновской длины волны (так как $\sigma \approx 1$ ). Благодаря эффективной перенормировке взаимодействия за счет множителя $\cos (\sigma x)$ снимаются парадоксы радиационного трения и исключаются расходимости при вычислении радиационных эффектов.

Анализ следствий из основного результата данной работы - формул (49) - выходит за рамки данной публикации. Здесь мы отметим только принципиально квантовый характер теории радиационного трения и отсутствие парадоксов, а именно рассмотрим вклад в радиационное трение первого слагаемого в (15) с учетом (49) в простейшем первом порядке малости по постоянной тонкой структуры. В этом случае для коэффициента трения в собственной системе координат можно воспользоваться выражением (48). 
Рассмотрим подробнее поведение коэффициента трения при частотах $\omega<\omega_{0} / 2$. В этой области можно воспользоваться аппроксимацией $\operatorname{arctg} x \approx x$. В результате из (48) получим

$$
\gamma(\tau)=\frac{4 \alpha}{3 \pi} \int_{0}^{\infty} d x \cos (\sigma x) \cos \left(\omega_{0} \tau\left(x+\frac{x^{2}}{2}\right)\right) \eta(\tau) .
$$

Нетрудно вычислить зависимость коэффициента радиационного трения от частоты. Для действительной части имеем

$$
\gamma^{\prime}(\omega)=\operatorname{Re} \int_{-\infty}^{\infty} d \tau e^{i \omega \tau} \gamma(\tau)=\frac{2}{3} \frac{e^{2}}{m c^{3}} \frac{1}{\sqrt{1+2|\omega| / \omega_{0}}} \cos \left(\sigma \frac{\omega}{\omega_{0}} \frac{2}{1+\sqrt{1+2|\omega| / \omega_{0}}}\right) .
$$

Заметим, что зависимость $\gamma^{\prime}(\omega)$ имеет принципиально квантовый характер. Действительно, входящее в данное выражение отношение $\omega / \omega_{0}=\hbar \omega / m c^{2}$ содержит в явном виде постоянную Планка. При $\hbar \rightarrow 0$ выражение $(50)$ переходит в классическое (27), из которого вытекают известные парадоксы.

Выясним физический смысл полученного выражения (50), в частности, покажем, что введенный выше параметр $\tau^{*}$ есть характерное время запаздывания взаимодействия. Для наглядности рассмотрим область более низких частот и, следовательно, малых передаваемых импульсов, когда слагаемым с $x^{2}$ можно пренебречь, а коэффициент трения (50) записать в виде

$$
\gamma(\tau)=\frac{4 \alpha}{3 \pi} \int_{0}^{\infty} d x \cos (\sigma x) \cos \left(\omega_{0} \tau x\right) \eta(\tau)
$$

Интегрирование по волновому вектору приводит к выражению

$$
\gamma(\tau)=\frac{2}{3} \alpha\left\{\delta\left(\omega_{0}\left(\tau-\tau^{*}\right)\right)+\delta\left(\omega_{0}\left(\tau+\tau^{*}\right)\right)\right\} \eta(\tau)=\frac{2}{3} \frac{\alpha}{\omega_{0}} \delta\left(\tau-\tau^{*}\right),
$$

из которого вытекает следующая зависимость коэффициента радиационного трения от частоты:

$$
\gamma(\omega)=\frac{2}{3} \frac{\alpha}{\omega_{0}} e^{i \omega \tau^{*}}=\frac{2}{3} \frac{e^{2}}{m c^{3}} e^{i \omega \tau^{*}} .
$$

С учетом выражения (52) линейная восприимчивость (30) запишется следующим образом:

$$
\chi(\omega)=-\frac{1}{m \omega^{2}}\left(1+i \frac{2 \alpha}{3} \frac{\omega}{\omega_{0}} e^{i \omega \tau^{*}}\right)^{-1} .
$$

Легко видеть, что это выражение не имеет полюсов в верхней полуплоскости комплексной переменной $\omega$ и, таким образом, удовлетворяет принципу причинности. В то же время коэффициент радиационного трения, который можно получить из классической формулы Абрагама-Лоренца (1),

$$
\gamma^{0}(\omega)=\frac{e^{2}}{m c^{3}}
$$

дает полюс в выражении для восприимчивости (30) в верхней полуплоскости $\omega$, что и приводит к парадоксу самоускорения в классической теории радиационного затухания. 
Из формулы (53) для линейной восприимчивости следует уравнение движения электрона во внешнем электрическом поле:

$$
\ddot{r}_{j}(t)-\gamma_{0} \dddot{r}_{j}\left(t-\tau^{*}\right)=\frac{e}{m} E_{j}(t) .
$$

Поскольку радиационная сила трения "включается" с опозданием на время $\tau^{*}$, приближенно можно считать, что в течение этого времени электрон движется только под действием внешнего поля. Следовательно, на этом промежутке времени мы имеем уравнение движения вида

$$
m \ddot{r}_{j}(t)=e E_{j}(t)
$$

На бо́льших промежутках времени влияние радиационной силы трения можно учесть, воспользовавшись ограниченным во времени уравнением движения

$$
\dddot{r}_{j}(t)=\frac{1}{m} \frac{d}{d t} e E_{j}(t)
$$

тогда для больших промежутков времени мы получим хорошо известное уравнение, применяемое для практических вычислений, но с некоторым принципиальным отличием:

$$
\ddot{r}_{j}(t)=\frac{e}{m} E_{j}(t)+\frac{e^{2}}{m c^{3}} \frac{d}{d t} e E_{j}\left(t-\tau^{*}\right) .
$$

Согласно полученному уравнению, электрон взаимодействует с электрическим полем с опозданием на время прохождения светом комптоновской длины волны. Это явление есть следствие эффекта нелокальности релятивистского квантового электрона, и оно полностью отсутствует в классической теории.

\section{6. ЗАКЛЮЧЕНИЕ}

В настоящей работе предложено принципиально важное обобщение установленного в работе [28] эффекта квантовой нелокальности, заключающегося в запаздывании взаимодействия между электроном и собственным полем излучения. В работе учтены флуктуации электронной плотности электрона Дирака, имеющие принципиально релятивистский квантовый характер, и определен асимптотический вклад больших передаваемых импульсов в эффект запаздывания взаимодействия между электроном Дирака и полем излучения с учетом обоих механизмов радиационного трения реакции собственного поля излучения и воздействия на электрон флуктуационного поля электромагнитного вакуума. Показано, что эффект запаздывания взаимодействия (квантовой нелокальности) исключает парадоксы самоускорения и нарушения причинности, имеющие место в классической теории.

Найдена зависимость коэффициента радиационного трения от частоты с учетом квантовых эффектов. Установленный эффект учитывает вклад в запаздывание взаимодействия всех импульсов, передаваемых от излучения к электрону. В области малых частот и, соответственно, малых передаваемых импульсов запаздывание взаимодействия является следствием того, что можно пренебречь быстрыми осцилляциями дисперсии приращения координаты в выражении для коэффициента радиационного трения. Вклад больших передаваемых импульсов в запаздывание взаимодействия получается в результате анализа асимптотического выражения для 
коэффициента трения при $k \gg k_{0}$. Значение времени запаздывания с большой точностью равно времени прохождения светом комптоновской длины волны $\lambda_{c}$.

Доказательство эффекта квантовой нелокальности приобретает особую значимость, так как при учете запаздывания взаимодействия автоматически исключаются расходимости и отпадает необходимость использования процедуры перенормировок при вычислении таких фундаментальных эффектов квантовой электродинамики, как смещение Лэмба и аномальный магнитный момент электрона. Исключение расходимостей в рамках простейшей модели статистической квантовой электродинамики позволяет надеяться на возможность распространения эффекта квантовой нелокальности на последовательную полевую теорию. Некоторые исходные положения флуктуационно-диссипационной квантовой электродинамики изложены в трудах конференций [27], [32]. В полевой теории запаздывание взаимодействия между зарядом и полем излучения также может быть связано с флуктуациями электронной плотности. При этом из соображений размерности мы получаем, что есть всего лишь одно характерное время: $\tau_{0}=\lambda_{c} / c$.

Таким образом, одночастичная квантовая электродинамика имеет достаточно широкую область применения [33], в частности, на ее основе можно исследовать широкий спектр радиационных эффектов, например радиационное трение, смещение Лэмба и аномальный магнитный момент электрона. Если перейти к более строгому описанию радиационных эффектов и учесть взаимодействие электромагнитного и электрон-позитронного вакуумов, то и в этом случае благодаря слабости их взаимодействия гауссова статистика для потенциалов вакуумного поля с большой степенью точности сохраняется (строгое физическое обоснование того, что негауссовость в вакуумных процессах является слабой, следует из нелинейных флуктуационно-диссипационных теорем). При этом функция Грина фотона изменится за счет поляризации электрон-позитронного вакуума и может быть вычислена лишь на основе полевой теории. Форма стохастических уравнений при этом останется прежней, и с большой точностью сохранится численное значение параметра нелокальности, определяющего перенормировку функции Грина фотона за счет эффекта запаздывания взаимодействия.

Приведенные выше физические соображения применимости одночастичной квантовой электродинамики (КЭД) согласуются с принципом соответствия. Применительно к КЭД принцип соответствия гласит следующее: полевая квантовая теория, являясь более общей теорией, включает в себя как предельный случай одночастичную КЭД. В то же время полевая теория строится на основе одночастичной релятивистской теории Дирака с привлечением принципа тождественности. Соответственно, одночастичная КЭД при $\hbar \rightarrow 0$ переходит в классическую электродинамику. Согласно принципу соответствия эффект квантовой нелокальности, установленный в одночастичной квантовой электродинамике, может быть перенесен в полевую теорию с помощью перенормировки функции Грина фотона.

\section{ПРИЛОЖЕНИЕ}

Для вычисления унитарного оператора $e^{-i B}$ введем фиктивное уравнение Шредингера

$$
-i \frac{\partial \psi}{\partial \theta}=\left(F_{0}+V\right) \psi(\theta)
$$

где $\psi$ - некоторая функция, $\theta$ играет роль временно́й переменной, $F_{0}=\mathbf{k} \Delta \mathbf{r}-$ оператор свободной эволюции, $V=\mathbf{k r}\left(t_{1}\right)$ - оператор взаимодействия. Решение 
этого уравнения записывается в виде

$$
\psi(\theta)=e^{i\left(F_{0}+V\right) \theta} \psi(0) .
$$

Наша задача - найти коэффициент радиационного затухания электрона во внешних полях, меняющихся во времени медленно, т.е. с частотой $\omega<\omega_{0}=m c^{2} / \hbar$. Поэтому для вычисления оператора $B$ будем использовать асимптотические по времени слагаемые. Из выражения (35) видно, что в случае $\omega_{0}\left(t-t_{0}\right)=\omega_{0} \tau>1$ оператор свободной эволюции $F_{0}=\mathbf{k}\left(\mathbf{r}(t)-\mathbf{r}\left(t_{1}\right)\right)$ равен

$$
F_{0}=\frac{c^{2} \mathbf{k p}}{H} \tau, \quad H=c \boldsymbol{\alpha} \mathbf{p}+\beta m c^{2} .
$$

Уравнение (П.1) нетрудно записать в представлении взаимодействия:

$$
-i \frac{\partial \widetilde{\psi}}{\partial \theta}=\widetilde{V}(\theta) \widetilde{\psi}(\theta)
$$

где

$$
\widetilde{\psi}(\theta)=e^{-i F_{0} \theta} \psi(\theta), \quad \widetilde{V}(\theta)=e^{-i F_{0} \theta} V e^{i F_{0} \theta} .
$$

Заметим, что в импульсном представлении оператор взаимодействия имеет вид $V=i \hbar \mathbf{k} \nabla_{\mathbf{p}}$, следовательно,

$$
\widetilde{V}(\theta)=V+e^{-i F_{0} \theta} i \hbar \mathbf{k} \frac{\partial}{\partial \mathbf{p}} e^{i F_{0} \theta} \equiv V+U(\mathbf{p}, \theta),
$$

и уравнение (П.4) можно переписать в виде

$$
-i \frac{\partial \widetilde{\psi}}{\partial \theta}=(V+U(\mathbf{p}, \theta)) \widetilde{\psi}(\theta) .
$$

Особенность вычисления производной в (П.6) обусловлена зависимостью оператора свободной эволюции $F_{0}$ от матриц Дирака. Из перестановочных соотношений для последних следует, что $F_{0}^{2}=\widetilde{F}_{0}^{2}$, где $\widetilde{F}_{0}=\left(c^{2} \mathbf{k p} / \widetilde{H}\right) \tau$, т.е. $F_{0}$ не содержит матриц Дирака. Поэтому можно записать следующее:

$$
e^{i F_{0} \theta}=\cos \left(\widetilde{F}_{0} \theta\right)+i \frac{F_{0}}{\widetilde{F}_{0}} \sin \left(\widetilde{F}_{0} \theta\right) .
$$

Воспользовавшись этим результатом и явным видом оператора свободной эволюции (П.3), для оператора $U(\mathbf{p}, \theta)$ получим следующее выражение:

$$
U(\mathbf{p}, \theta)=-\frac{\tau \hbar c^{2} k^{2} \theta}{H}+\frac{\tau \hbar c^{4}(\mathbf{k p})^{2} \theta}{H^{3}} .
$$

Используем в уравнении (П.7) представление взаимодействия и будем искать решение в виде

$$
\widetilde{\psi}(\theta)=e^{i V(\theta-1)} \varphi(\theta) .
$$

В этом случае для функции $\varphi(\theta)$ получаем уравнение

$$
-i \frac{\partial \varphi}{\partial \theta}=\widetilde{U}(\mathbf{p}, \theta) \varphi(\theta),
$$


где

$$
\widetilde{U}(\mathbf{p}, \theta)=e^{-i V(\theta-1)} U(\mathbf{p}, \theta) e^{i V(\theta-1)}=e^{-i \mathbf{k r}\left(t_{1}\right)(\theta-1)} U(\mathbf{p}, \theta) e^{i \mathbf{k r}\left(t_{1}\right)(\theta-1)} .
$$

Замечаем, что в координатном представлении оператор импульса $\mathbf{p}=-i \hbar \nabla$. Нетрудно доказать следующее равенство:

$$
\widetilde{U}(\mathbf{p}, \theta)=U(\mathbf{p}+\hbar \mathbf{k}(\theta-1), \theta) .
$$

Поскольку оператор $\widetilde{U}(\mathbf{p}, \theta)$ не коммутирует с $\widetilde{U}\left(\mathbf{p}, \theta_{1}\right)$ при $\theta \neq \theta_{1}$, решение уравнения (П.10) необходимо записать в соответствии с теорией $S$-матрицы:

$$
\varphi(\theta)=T \exp \left\{i \int_{0}^{\theta} d \theta_{1} \widetilde{U}\left(\mathbf{p}, \theta_{1}\right)\right\} \varphi(0)=S(\theta) \varphi(0),
$$

где $T$ - оператор хронологического упорядочения по параметру $\theta$.

Найдем связь между исходной функцией $\psi(\theta)$ и найденной $\varphi(\theta)$. Из (П.5) и (П.9) получим

$$
\psi(\theta)=e^{i F_{0} \theta} e^{i V(\theta-1)} \varphi(\theta)
$$

Используя (П.12) и (П.13), для уравнения (П.1) при $\theta=1$ имеем следующее решение: $\psi(1)=e^{i F_{0}} S(1) e^{i V} \psi(0)$, при этом из общего решения (П.12) следует, что $\psi(1)=$ $e^{i\left(F_{0}+V\right)} \psi(0)$. Приравнивая эти два результата, находим, что $e^{i\left(F_{0}+V\right)}=e^{i F_{0}} S(1) e^{i V}$, или, в исходных обозначениях,

$$
e^{i \mathbf{k r}(t)}=e^{i \mathbf{k} \Delta \mathbf{r}} S(1) e^{i \mathbf{k r}\left(t_{1}\right)}
$$

В результате получаем искомое произведение экспонент (32):

$$
e^{i \mathbf{k r}(t)} e^{-i \mathbf{k r}\left(t_{1}\right)}=e^{i \mathbf{k} \Delta \mathbf{r}} T \exp \left\{i \int_{0}^{1} d \theta U(\mathbf{p}+\hbar \mathbf{k}(\theta-1), \theta)\right\}
$$

Из формул (П.8) и (П.11) следует слабая некоммутативность операторов $\widetilde{U}(\mathbf{p}, \theta)$ при разных $\theta$, поэтому с большой точностью можно пренебречь взятием операции хронологического упорядочения в (П.14). Заметим, что для бесспиновой релятивистской частицы операторы $\widetilde{U}(\mathbf{p}, \theta)$ при различных значениях $\theta$ коммутируют друг с другом и $S$-матрица вычисляется точно.

Используя (П.8) и произведя интегрирование в собственной системе отсчета электрона, для правой части (П.14) получаем

$$
\begin{aligned}
& T \exp \left\{i \int_{0}^{1} d \theta U(\hbar \mathbf{k}(\theta-1), \theta)\right\}= \\
& \quad=\exp \left(-\frac{i \omega \tau}{2}\left\{\beta \frac{k}{k_{0}} \operatorname{arctg}\left(\frac{k}{k_{0}}\right)+\frac{\mathbf{k} \boldsymbol{\alpha}}{k_{0}}-\frac{\mathbf{k} \boldsymbol{\alpha}}{k} \operatorname{arctg}\left(\frac{k}{k_{0}}\right)\right\}\right) .
\end{aligned}
$$

Благодарности. Авторы выражают признательность Ю. Г. Шондину за полезные обсуждения ряда вопросов, затронутых в настоящей работе. 


\section{Список литературы}

[1] M. Abraham, Theorie der Elektrizität. II. Elektromagnetische Theorie der Strahlung, Teubner, Leipzig, 1905; Г. А. Лоренц, Теория электронов, Гос. издат. техн.-теор. лит., M., 1953.

[2] P. A. M. Dirac, Proc. Roy. Soc. Lond. Ser. A, 167:929 (1938), 148-169.

[3] Л. Д. Ландау, Е. М. Лифшиц, Теоретическая физика. Т. II. Теория поля, Наука, М., 1973.

[4] Дж. Джексон, Классическая электродинамика, Мир, М., 1965.

[5] В. Л. Гинзбург, Теоретическал физика и астрофизика, Наука, М., 1981.

[6] Г.Ф. Ефремов, ЖЭТФ, 110 (1996), 1629-1640.

[7] Г.Ф. Ефремов, ЖЭТФ, 114:5 (1998), 1661-1671.

[8] П. О. Казинский, А. А. Шарапов, ТМФ, 143:3 (2005), 375-400.

[9] Б. П. Косяков, ТМФ, 119:1 (1999), 119-135.

[10] F. Rohrlich, Phys. Lett. A, 283:5-6 (2001), 276-278; 295:5-6 (2002), 320-322.

[11] В. В. Лидский, ТМФ, 143:1 (2005), 112-130.

[12] R. P. Feynman, Phys. Rev., 74:8 (1948), 939-946.

[13] G. W. Ford, J. T. Lewis, R. F. O'Connell, Phys. Rev. Lett., 55:21 (1985), 2273-2276.

[14] P. M. Barone, A. O. Caldeira, Phys. Rev. A, 43:1 (1991), 57-63.

[15] V. Hakim, V. Ambegaokar, Phys. Rev. A, 32:1 (1985), 423-434.

[16] A. I. Nikishov, V.I. Ritus, ЖЭТФ, 114:3 (1998), 777-785.

[17] A. Komech, Phys. Lett. A, 241:6 (1998), 311-322.

[18] A. C. R. Mendes, F. I. Takakura, Phys. Rev. E, 64:5 (2001), 056501.

[19] T. Petrosky, G. Ordonez I. Prigogine, Phys. Rev. A, 68:2 (2003), 022107.

[20] A. Yu. Smirnov, J. Phys. A, 30:4 (1997), 1135-1141.

[21] A. Yu. Smirnov, Phys. Rev. E, 56:2 (1997), 1484-1489.

[22] Г.Ф. Ефремов, В. А. Казаков, Изв. вузов. Сер. радиофиз., 22 (1979), 453.

[23] Г.Ф. Ефремов, А. Ю. Смирнов, ЖЭТФ, 80 (1981), 1071.

[24] Н. Н. Боголюбов, Н. Н. Боголюбов (мл.), ТМФ, 43:1 (1980), 3-17.

[25] J. Schwinger, J. Math. Phys., 2 (1961), 407-432.

[26] I. R. Senitzky, Phys. Rev., 119:2 (1960), 670-679; 124:3 (1961), 642-648.

[27] G.F. Efremov, M. A. Novikov, L. G. Mourokh, "Fluctuation-dissipation quantum electrodynamics", The Present Status of the Quantum Theory of Light, Fund. Theories Phys., 80, eds. S. Jeffers, S. Roy, J.-P. Vigier, G. Hunter, Kluwer, Dordrecht, 1997, 97.

[28] Г. Ф. Ефремов, В. В. Шарков, ЖЖЭТФ, 125:2 (2004), 195-204.

[29] П. А. М. Дирак, Приницпы квантовой механики, Физматгиз, М., 1960.

[30] А. А. Абрикосов, Л. П. Горьков, И. Е. Дзялошинский, Методы квантовой теории поля в статистической физике, Физматгиз, М., 1962.

[31] Г. Ф. Ефремов, ЖЭТФ, 55 (1968), 2322.

[32] G. F. Efremov, M. A. Novikov, V. V. Ivanov, A. G. Efremov, "Causality and nonlocality in problem of radiative damping of electron", Causality and Locality in Modern Physics, Fund. Theories Phys., 97, eds. G. Hunter, S. Jeffers, J.-P. Vigier, Kluwer, Dordrecht, 1998, 87-95.

[33] Дж. Д. Бьеркен, С. Д. Дрелл, Релятивистская квантовая теория. Т. I. Релятивистская квантовая механика, Наука, М., 1978.

Поступила в редакцию 30.11 .2007 , после доработки 27.06.2008 